\title{
Early supersymmetry searches with ATLAS detector at LHC.
}

\author{
U. De Sanctis on behalf of the ATLAS Collaboration \\ Università di Milano - Dipartimento di Fisica and Istituto Nazionale di Fisica \\ Nucleare - Sezione di Milano, Via Celoria 16, I-20133 Milan, Italy
}

Summary. In this paper, a brief overview of the principal strategies for Supersymmetry searches with ATLAS detector at LHC is presented. The aim is to evaluate the ATLAS discovery potential in inclusive channels with early data with particular attention to the more interesting techniques to estimate the principal SM backgrounds using real data.

\section{Generalties on Supersymmetry}

One of the main purposes of LHC is the search for physics beyond the Standard Model (SM). In this framework, Supersymmetry (SUSY) plays an important role, because it's one of the most popular and credited candidates to extend the SM[1]. It introduces a new symmetry that for each SM boson predicts a fermionic super-partner and vice versa. Following this symmetry, one can classify all SUSY particles foreseen by the theory dividing them into: scalar fermions (called sleptons and squarks) and gauginos (called Winos, Binos, Zinos, photinos and gluinos). Higgs sector is composed by two doublets of fermions (in order to avoid triangular anomalies) that give origine to five physical scalar bosons, and four fermionic partners called Higgsinos (two neutral and two charged).

While the gluino is a mass eigenstate, Higgsinos and the other gauginos mix giving four charged mass eigenstates called Charginos and four neutral mass eigenstates called Neutralinos. In the R-parity (quantum number that has value +1 or -1 respectively for SM and SUSY particles) conserving models, the lightest neutralino is the LSP (Lightest Supersymmetric Particle) that provides a suitable candidate for Dark Matter because it's stable, neutral and weakly interacting.

Nevertheless, SUSY has not been discovered yet, and this means that this symmetry must be broken (electron and s-electron have different mass). Hence, one needs to add in the lagrangian some terms breaking Supersymmetry in order to remove mass degeneracies between particles and their super-partners. 
The final number of free parameters needed for MSSM (Minimal Supersymmetric Standard Model) is then 105, including mass terms, couplings, mixing angles and CP-violation phases. Because of this large number of free parameters, a more constrained frameworks are often used at LHC in order to develop analysis strategies. In this paper the focus will be on strategies for mSUGRA scenario.

In section 2 an overview of the ATLAS performances for inclusive SUSY searches and discovery potential in mSUGRA scenario are reported, while in section 3 the most important techniques to estimate SM backgrounds for SUSY analysis starting from data are shown.

\section{Early SUSY searches and discovery potential in ATLAS in mSUGRA model.}

\subsection{Introduction on mSUGRA}

The mSUGRA model is characterised by gravity mediated SUSY-breaking[2], and foresees only five independent parameters to define SUSY sector: the common gaugino mass $m_{1 / 2}$, the common scalar mass $m_{0}$, the common trilinear gauge coupling $A_{0}$ at some high unification scale, the ratio of the vacuum expectation values of the two Higgs doublets $\tan \beta$ and the sign of the Higgsino mixing parameter $\mu$. The top mass can be traited like a sixth independent parameter because it strongly affects the value of physical quantities.

All the analysis in this section are performed in this framework with the addition of the R-parity conservation.

In order to perform detailed studies of SUSY discovery potential for the ATLAS detector, specific sets of values of the mSUGRA space parameters have been chosen (benchmark points) [3], taking into account the constraints arising from experimental data (direct searches on Higgs and SUSY at LEP, precision tests at B-factory), theoretical reasons (request of electroweak symmetry breaking mechanism) and cosmological data (compatibility of abundance of cold Dark Matter in the Universe with relic density of lightest neutralinos). [5] These constraints are satisfied in four relatively narrow regions of mSUGRA space, characterized by annihilation processes between SUSY particles that make relic density compatible.[6]

\subsection{Analysis strategies}

Inclusive searches essentially consist in a check for any significant deviation from SM predictions in channels characterized by a particular signature. If SUSY exists at the scale of about $1 \mathrm{TeV}$ (as requested to solve hierarchy problem), this kind of searches needs a relatively small integrated luminosity, but are strongly dependent on a very good knowledge of SM processes at that energy scale (never explored until now) and on an understanding of detector's 
performances. Hence, the goal of this kind of searches is to find a statistically significant deviation from SM predictions in some characteristic kinematical variable and to do an approximate estimation of the SUSY mass scale. More difficult in this case will be the possibility to strongly constrain model's parameters.

At LHC, in the R-parity conserving framework, SUSY particles will be produced in pairs and coloured states (scalar quarks and gluinos) are expected to dominate the production cross section; their decay chains into LSP, which escapes detection, produce an excess of events with multijets, missing energy and isolated leptons final states compared to the SM expectations.

Probably this framework is only a simplified approximation to the rich SUSY phenomenology but is nearly completely determined at hadron-collider by only two parameters $\left(m_{0}\right.$ and $\left.m_{1 / 2}\right)$ which determine masses and production cross-sections of the strongly interacting sparticles (gluinos and squarks). Inclusive searches for SUSY therefore concentrate on channels containing jets, $n$ leptons and $E_{T}^{M I S S}$, with the required number of leptons being varied depending on the desired signal-to-background ratio and hence sensitivity to systematic uncertainties in background rate.

The signal events from mSUGRA models in different points of $m_{0}-m_{1 / 2}$ plane and principal background sources coming from $t \bar{t}, \mathrm{~W}+\mathrm{n}$ jets, $\mathrm{Z}+\mathrm{n}$ jets and QCD events $(q \bar{q}$ production, except $t \bar{t})$ are generated, while parametrized detector simulation is carried out using ATLFAST[4].

A first kind of analysis that can be developped consists on choosing a set of kinematical variables for a specific channel jets $+E_{T}^{M I S S}+n$ leptons (with $n=0,1,2,3$ ), on optimizing cuts and on plotting it for signal SUSY events and for SM background events. A different behaviour of the shape of the distributions can show an eventual significant excess of events that SM does not foresee.

The most promising channel for an early discovery is the one with Jets $+E_{T}^{M I S S}$ +1 lepton, because the request of one isolated lepton reduces sensibly the QCD background (difficult to estimate, especially with early data, for its high jets multiplicity) leaving $t \bar{t}$ and $\mathrm{W}+$ jets events as the dominant backgrounds (possible to estimate directly from data, see next chapter). The plot of Fig.1 represents the effective mass distribution (i.e. the sum of missing energy and transverse momenta $P_{T}$ of jets in the event) for SUSY events and different SM backgrounds: for high value of this kinematical variable (more than $1.5 \mathrm{TeV}$ ) is possible to distinguish the different contributions coming from SUSY with respect to SM events. Calculating then the significance as explained above, one can evaluate the discovery potential of this channel

The set of cuts applied to obtain this plot are the following:

$E_{T}^{M I S S}>100 \mathrm{GeV}$; Hardest jet with $P_{T}>100 \mathrm{GeV}$ and 4 jets with $P_{T}>$ $50 \mathrm{GeV}$; Transverse sfericity $S_{T}>0.2$ (this variable depends on the angular distribution of the jets in the event); 1 isolated muon or electron with $P_{T}>10 \mathrm{GeV}$ 


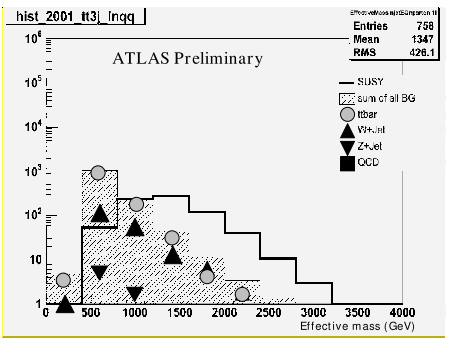

Fig. 1. Effective mass distribution, for an integrated luminosity of $10 \mathrm{fb}^{-1}$. Solid line reprents SUSY signal, dashed area represents the sum of the principal SM backgrounds: $t \bar{t}$ events (dots), $\mathrm{W}+\mathrm{n}$ jets and $\mathrm{Z}+$ jets events(triangles) and $\mathrm{QCD}$ events (boxes).

\section{Background estimation from data}

In order to have a possibility to claim a discovery of SUSY, one needs a very good knowledge both of SM background events and detector performances. Both arguments will be rapidly traited on this section. There are different techniques to estimate background from data; I will explain briefly some examples of the estimation of the SM backgrounds for SUSY analysis.

As above, the more important variable to discover SUSY is missing energy, hence one must estimate very carefully the contribution coming from SM events. For example, in $\mathrm{Z}+$ jets events, the big contribution to missing energy comes from $Z \rightarrow \nu \nu$ decays; one can estimate it using $Z \rightarrow$ ee decays, appliying the same cuts as the analysis, substituting the transverse energy for the 2 electrons reconstructed with tranverse missing energy and then rescaling the contribution for the different branching ratios between the two channels.

Another useful strategy starts on choosing 2 uncorrelated variables, for example the top mass and $E_{T}^{M I S S}$. One selects events with invariant mass lepton-jet $m(l \nu j)$ in top mass window (for ex. $175 \pm 20 \mathrm{GeV}$ ) (using $\mathrm{W}$ mass to constraint lepton and missing energy) and estimates combinatorial background in this window using sideband subtraction, i.e. events with $m(l j)$ out of the top mass window choosen above (for ex. in the interval 195-235 GeV). For the events choosen in this way one can be pretty sure that they come from SM processes and that the SUSY contribution is very small. Looking then to the missing energy distribution of these events and normalizing it to the overall missing energy distribution in the low $E_{T}^{M I S S}$ region where SUSY is expected to give small contributions (for ex. in the interval 100-200 GeV), one can extrapolate the shape of the missing energy for "top events" in the high $E_{T}^{M I S S}$ region and then estimate the contribution of $t \bar{t}$ events in that region, where SUSY should be strongly present.

Fig. 2 shows the technique explained above in the missing energy distribution. 


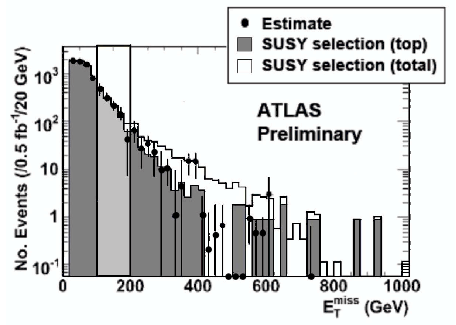

Fig. 2. Missing energy distribution distribution, for an integrated luminosity of 0.5 $\mathrm{fb}^{-1}$. White area represents the overall distribution of the event, dark area represents missing energy for $t \bar{t}$ events, while dots represents the estimation of $t \bar{t}$ contribution to the overall distribution in the high $E_{T}^{M I S S}$ region. In evidence also the low $E_{T}^{M I S S}$ region (100-200 $\mathrm{GeV}$ window) where the two distributions are normalized as explained in the text.

\section{Conclusions}

If Supersymmetry exists at the electroweak scale, the initial discovery depends on how quickly we can understand the systematics coming from the detector and the background knowledge. Studies in inclusive channels have been presented, in the framework of mSUGRA model and GMSB model with R-parity conservation. The more interesting techniques of SM background estimation from data have already been presented.

In mSUGRA models, the more promising channel for a quick discovery is the signature Jets $+E_{T}^{M I S S}+1$ lepton, once the backgrounds of SM (especially $t \bar{t}$ events) will be understood and carefully estimated from data. In these conditions the SUSY discovery needs less than $1 \mathrm{fb}^{-1}$ if s-particle masses are of order of $1 \mathrm{TeV}$.

\section{References}

1. Y.Gol'fand, E.Likthman: JETP Lett 13 (1971) 323;

D.Volkov, V.Akulov: Phys. Lett. B46 (1973) 109;

J.Wess, B.Zumino: Nucl. Phys. B70 (1974) and B78 (1975) 1.

2. H.P.Niles: Phys Rev. 110 (1984) 1;

A.H.Chamseddine, R.Arnowitt et P.Nath: Phys. Rev. Lett. 49, 970 (1982).

3. The ATLAS Collaboration: ATLAS Detector and Physics Performance Technical Design Report CERN/LHCC 99-14 (1999).

4. E.Rochter-Was, D.Froidevaux et L.Poggioli: ATLAS Note ATL-PHYS-98131.

5. J.R.Ellis et al. : Phys. Lett. B565 (2003) 176;

H.Baer et C.Balasz: J. Cosmol. Astr. Phys. 0305 (2003) 006.

6. U.De Sanctis : Il Nuovo Cimento VOL.121 B, N.8. 